\title{
Una canción inédita de Salomón Usque
}

Jordi CANALS PIÑAS *

Università degli Studi di Trento

Muy escasas son las noticias biográficas que de Salomón Usque han llegado hasta nosotros. Sabemos que era descendiente de judíos conversos portugueses, muchos de los cuales a partir de 1536 abandonaron masivamente el territorio lusitano dando lugar a colonias importantes en el puerto de Amberes y acto seguido en Ferrara, la capital del ducado Estense en cuya tolerante corte muchos de ellos volvieron abiertamente a la fe de sus antepasados. Él mismo corrobora tal origen al firmar con el seudónimo humanístico de Salusque Lusitano en frontispicio una parte de los ejemplares de su traducción castellana del Canzoniere de Petrarca (Venecia 1567) ${ }^{1}$. Sospechamos que fue hijo de Abraham Usque, asentado en Ferrara y con imprenta en Vignatagliata, en el barrio de San Romano, de donde salieron los ejemplares de la Biblia de Ferrara (Ferrara

\footnotetext{
*Jordi.Canals@lett.unitn.it

${ }^{1}$ De los sonetos, canciones, mandriales y sextinas del gran poeta y orador Francisco Petrarca [sólo primera parte del Canzoniere], trad. Salomón Usque Hebreo [Salusque Lusitano] (Venezia: Nicolò Bevilacqua, 1567). Vid. J. CANals, Salomón Usque, traductor del "Canzoniere» de Petrarca. Estudio y edición crítica (Bellaterra: Universitat Autònoma de Barcelona, 2001) [tesis doctoral].
}

Sefarad 64 (2004) págs. 3-25

(C) CSIC

ISSN 037-0894 
1553) ${ }^{2}$ y cuya actividad tipográfica continuó hasta el invierno de 1557-1558, época tras la cual se trasladó a Venecia y acto seguido tal vez a Turquía ${ }^{3}$.

Tanto Abraham como Salomón Usque entraron en relación con el círculo de la influyente judía portuguesa Gracia Nasi alias Beatriz de Luna ${ }^{4}$, que desde fines de 1548 y hasta 1553 , fecha en la que se trasladó definitivamente a Constantinopla, residió junto con su hermana Brianda en Palazzo Magnanini de Ferrara. Un soneto de Duarte Gómez precede a la mencionada traducción del Canzoniere y allí, el que todavía era agente comercial en Italia de la rica heredera del patrimonio de los Mendes, pondera la tarea que Salomón Usque ha llevado a cabo.

Nos consta el paso de Salomón por la República Serenissima ya hacia 1560 , cuando pudo haberse escenificado por vez primera, en

${ }^{2}$ C. Roth, que no tenía ninguna duda de tal paternidad; afirma en «Salusque Lusitano. An Essay in Disentanglement» [1931], en Gleanings. Essays in Jewish History. Letters and Art (New York 1967) pág. 191: «Among the assistants at the printing house, for the production of the Hebrew books, was Abraham Usque's son, Salomon, named after his grandfather». Conocemos la ubicación del taller tipográfico ferrarés del «hebreus portugensis impressor» gracias a un documento notarial fechado en 1554 y del que da noticia R. SEGRE, «La formazione di una comunità marrana: I portoghesi a Ferrara», en Storia d'Italia. Annali 11: Gli ebrei in Italia. I, ed. C. VIVANTI, (Torino 1996) pág. 817. Para una visión de conjunto remitimos a Introducción a la Biblia de Ferrara, ed. I. M. HASSÁN (Madrid 1994); vid. nuestra reseña en Romanistik in Geschichte und Gegenwart 2.2 (1996) págs. 223-225.

${ }^{3}$ Un documento notarial fechado en la ciudad lagunar el 17 de agosto de 1559 nos informa de que Abraham Usque adquirió caracteres hebraicos al tipógrafo Mazo de Parenzo. En dicho acto de compra figura una cláusula que compromete al nuevo propietario a no emplear los tipos en territorio veneciano, lo que es indicio de que el impresor portugués había decidido tal vez emigrar al Levante; vid. R. SEGRE, «La tipografia ebraica a Ferrara e la stampa della Bibbia (1551-59)», Italia Medioevale e Umanistica 35 (1992) págs. 328-329.

${ }^{4}$ Viuda del comerciante Francisco Mendes, muerto en 1543. Era tía y suegra de Yosef Nasi, que antes de su traslado a Ferrara la libró del arresto a la que había sido condenada en Venecia por prácticas de criptojudaísmo denunciadas por la propia hermana Brianda (vid. C. ROTH, Gli ebrei in Venezia (Roma: 1933) págs. 74 y 96). En torno a las hermanas Mendes-Nasi, señalamos la contribución de H. P. SALOMON y A. di Leone LEONI, «Mendes, Benveniste, de Luna, Micas, Nasi: The State of the Art», The Jewish Quarterly Review 88 (1998) págs. 135-211. 
la judería de Cannaregio, la tragedia L'Ester con motivo de la festividad de Purim. Así lo rememora, aunque con cierta imprecisión («Sessant'anni in circa sono»), el rabino Leone Modena en el prefacio a la edición del texto dramático que refundió y publicó en Venecia en $1619^{5}$.

Su nombre figura como tipógrafo en ediciones de obras judías impresas en Constantinopla en los años 1560-1561, algunas de las cuales junto a Abraham bar Samuel Ashkenazi, el cual había sido ayudante de Abraham Usque en la imprenta de Ferrara ${ }^{6}$. La ida a Constantinopla es dato que podría ponerse en relación con la mencionada compra de tipos hebraicos que en verano de 1559 Abraham Usque había adquirido en Venecia (v.s.n. 3), lo que corroboraría el vínculo directo con Salomón. En tal caso, a éste legaría no sólo el apellido, sino también sus directrices editoriales, pues las obras que editó en la capital otomana suponen una continuidad de la labor tipográfica ferraresa. Pero la de Salomón Usque debió de ser actividad efímera y poco lucrativa, ya que no tenemos constancia de que se imprimieran otros libros con su marca tipográfica ni en Constantinopla, ni en ningún otro lugar, más allá de 1561.

Sólo a inicios de 1595 el nombre del lusitano aparecerá de nuevo en Constantinopla como autor de un curioso informe escrito en italiano y

\footnotetext{
${ }^{5}$ Es edición de extremada rareza impresa por Giacomo Sarzina y de la que hay un ejemplar depositado en Biblioteca Marciana de Venecia (Signatura: Dramm. 698). El prefacio al que aludimos («L'Autore a' benigni lettori») se halla entre las páginas 8-11 y sigue a la dedicatoria a Sarra Copio Sullam Hebrea (pp. 3-6), fechada el 25 de febrero de 1618. V.q. Abramo A. PiatTelli, «Ester: L'unico dramma di Leon da Modena giunto fino a noi», Rassegna Mensile di Israele 34 (1968) págs. 163-172.

${ }^{6}$ Es dato que proporciona C. RoTH, «Salusque Lusitano. An Essay...», pág. 193, pero sin que facilite indicación de la fuente. Juntos editaron Judah Nathan Provençal, 'Or 'olam; tif'eret Yisrael [Luz del mundo, Gloria de Israel. Capitulo sobre la adhesión] (Constantinopla: ¿1560?) y Solomon Halevi Alkabez, Shoresh Yishai [Libro del linaje de Yishay] (Constantinopla: 1560-61); vid. C. RoTH, ibid., pág. 193, n. 38, y Y. H. Yerushalmi, «A Jewish Classic in the Portuguese Language», en Samuel Usque, Consolação às tribulações de Israel: Edição de Ferrara, 1553, vol. I (Lisboa 1989) pág. 122. En solitario imprimió Salomón Usque el libro bíblico de Rut; vid. G. BusI, Edizioni ebraiche del XVI ${ }^{\circ}$ secolo nelle biblioteche dell'Emilia Romagna (Bologna: 1987) § 178.
} 
vendido a Sir Edward Barton, que en esta fecha se hallaba acreditado como embajador de la Corona Británica en la Puerta Sublime ${ }^{7}$. El documento proporciona numerosos datos en torno al sultán Amurates III, cuya muerte acababa de producirse el 18 de enero de 1595, y da noticia detallada del acceso al trono de su primogénito, así como de las celebraciones oficiales con las que el nuevo sultán Mahmud III dio inicio a su gobierno ${ }^{8}$. Es el último documento del que tenemos noticia en el que aparezca el nombre del judío lusitano.

Salomón Usque es autor de poesías escritas, casi todas ellas en italiano. Si exceptuamos el soneto laudatorio a don Luis de Zúñiga y Requesens, futuro lugarteniente de don Juan de Austria en Lepanto, al frente de Comentarios del S. Alonso de Ulloa, de la guerra que el illustríssimo y valerosíssimo príncipe don Hernando Álvarez de Toledo, duque de Alva y capitán general del rey Don Felipe, nuestro señor, ha hecho contra Guillermo de Nausau, príncipe de Oranges (Venezia 1569) 9, la única composición de Usque que se llegó a imprimir es la Canzone sull'opera de' sei

\footnotetext{
${ }^{7}$ Fue el segundo embajador británico desde que el Reino Unido, interesado en comerciar directamente con el imperio otomano sin tener que someterse al monopolio veneciano, estrechó lazos con aquel en 1580. Es sugerente el panorama esbozado por H. G. Rosedale, Queen Elizabeth and the Levant Company (London 1904).

${ }^{8}$ Manuscrito depositado en The Public Record Office de Londres (Signatura: S.P. Turkey [S.P. 97] III/T3 [fols. 1-12]). Firmado por «Salomone Usche hebreo» en fecha del 2 de febrero de 1595, aunque la crónica de los acontecimientos abarca hasta el 5 de febrero de 1595. Fue reproducido y traducido al inglés por H. G. RoSEDALE, Queen Elizabeth, págs. 19-46. Editamos críticamente el documento en «Un informe otomano de Salomón Usque (1595)», Espacio, tiempo, forma [en prensa].

${ }^{9}$ Es una rara edición en español impresa por Domingo de Farris y de la que sólo conocemos el ejemplar depositado en The British Library de Londres (Signatura: 9405.cc.5); no la registra A. RUMEU DE ARMAS, Alfonso de Ulloa, introductor de la cultura española en Italia (Madrid 1973), en su inventario y descripción de obras de Ulloa. Trascribimos dicho soneto: «A quién podía encomendar su historia / l'Ulloa, para que más se loe y cante, / qu'al gran Comendador, Grand'Almirante / digno d'eterna y d'immortal memoria. / Aquéste da a Castilla assaz más gloria, / que la que a Roma César dio triunfante. / Y si como ha empeçado va adelante, / aun le dará del mundo la victoria. / Assí que heroicos hechos al heroa / se deven dedicar y en esto muestra / (como en lo más) su juizio el nuestro Ulloa. / El cual la pluma ya tiene en la diestra / para del Requessens don Luis famoso / con estilo escrevir muy sonoroso».
} 
giorni, incluida en la antología compilada por Cristoforo Zabata y titulada Nuova scelta di rime di diversi belli ingegni (Genova 1573) ${ }^{10}$. Es canción de nueve estancias dedicada a Carlo Borromeo, el futuro santo patrón de la ciudad de Milán ${ }^{11}$. En ella Usque describe, a lo largo de sus ciento cuarenta y cinco versos, la creación del cielo y de la tierra al hilo del tradicional relato bíblico: el poeta enumera, día a día y una tras otra, las creaciones que por obra de Dios brotan en el universo. Es descripción que Usque lleva a cabo de acuerdo con la cronología del primer libro del Pentateuco (Gén. 1:1-2:25) y en la que la narración de lo ocurrido en cada uno de los seis días de la creación se distribuye regularmente en las estrofas.

Es autor asimismo de dos sonetos que figuran como colofón del mencionado informe otomano y en los cuales evoca el asesinato de los hermanastros de Mahmud III, episodio central de la crónica que precede.

Quienes se han aproximado a la vida y obra de Salomón Usque no dan noticia de una composición que se halla en un códice manuscrito depositado en la Biblioteca Comunale Ariostea de Ferrara ${ }^{12}$, que incluye entre fols. 308r-315v una canción italiana de Salomón Usque que ha permanecido inédita hasta hoy.

\footnotetext{
${ }^{10}$ La composición de Usque se halla entre págs. 109-114; vid. Y. H. YeRUSHALMI, «A Jewish Classic in the Portuguese Language», pág. 122. Nosotros hemos accedido al poema a través de una reedición posterior: Scelta di rime di diversi eccellenti autori [...] Parte prima (Genova, s.t.: 1582) [prólogo de Cristoforo Zabata fechado el 16 de septiembre de 1582], págs. 116-122. Unas décadas más tarde, Torquato Tasso, tan vinculado a la corte de Ferrara, compondrá con idéntico tema Le sette giornate del mondo creato (1594), como nos recuerda Rossend ARQUÉS (UAB).

${ }^{11}$ Vid. la extensa nota de M. DE CERTAU, en DBI, s.v. Carlo Borromeo. No nos queda claro el propósito de Usque al dedicar la composición al arzobispo de Milán, por lo demás hostil a los marranos, como nos advierte R. SEGRE. Tal vez se dirige a él por considerarlo personaje influyente en un territorio bajo dominio español. Recuérdese que el Milanesado fue cedido en 1525 por la corona de Francia a Carlos $\mathrm{V}$, y que desde 1556, tras la batalla de Pavía, la gobernación del territorio está en manos de Felipe II.

${ }^{12}$ Ms. N.A.5, descrito por M. MESSINA, «Rime del XVI ${ }^{\circ}$ secolo in un manuscrito autografo di G.B. Giraldi Cinzio e di B. Tasso», La Bibliofilia 57 (1955) págs. 108147, quien ha demostrado que la transcripción en letra cursiva del códice es en su mayor parte de mano de Giambattista Giraldi Cinzio, profesor y secretario del duque de Este (pág. 110). No así estos folios, cuya caligrafía tampoco coincide con la de Bernardo Tasso, a quien se adscribe otra parte del manuscrito.
} 
Es poema encomiástico dirigido a Guidobaldo II Montefeltro (1514-1574), que a la muerte del padre Francisco Maria della Rovere, acaecida el 20 de octubre de 1538, le sucedió al frente del ducado de Urbino. La canción pudo haber sido escrita con motivo de una de las visitas oficiales del duque de Urbino a la corte estense. En tal caso la más probable parecería ser la de 1566, ocasión en la que el duque está acompañado por su segunda esposa Vittoria Farnese ${ }^{13}$, si no fuera, porque en estos años nuestro traductor parece hallarse ya muy lejos del ducado de Ferrara. La de 1548, cuando Guidobaldo II llega a la ciudad estense formando parte del séquito de Giulia della Rovere, futura consorte de Alfonso di Montecchio nos parece por otro lado demasiado temprana.

Si descartamos la relación con una visita oficial del duque de Urbino, la petición de auxilio por parte de Usque podría ponerse en relación con el proyecto de Guidobaldo II para potenciar el puerto de Pesaro, aprovechando la amenaza de embargo del tráfico marítimo levantino con Ancona a raíz de los autos de fe que tuvieron lugar en el emporio adriático en el año 1555. Tal proyecto naufragó de modo definitivo en 1556, y a partir de dicho año la presencia de judíos en el ducado de Urbino es poco más que simbólica ${ }^{14}$.

${ }^{13}$ En 1566 la visita de los duques sigue a la del heredero Francesco Maria della Rovere, el cual en fecha de 2 de enero había transitado por la corte estense en su camino hacia Madrid, capital en la que permanecería tres años al servicio imperial de Felipe II y a cuyo regreso de la Península Ibérica se estipularía el contrato del matrimonio del heredero del ducado de Urbino con Lucrezia d'Este (22 de diciembre de 1569). Más tardía aún es la del 22 de octubre de 1569 , fecha en la que se estipula el contrato de matrimonio entre Francesco Maria della Rovere y Lucrezia d'Este. No se mencionan otras visitas del duque de Urbino en los diarios de Carlo Olivi, Annali della città di Ferrara. Dalla sua prima origine fino al dominio delli ultimi Ducchi Estensi. I [1790], ms. de la Biblioteca Comunale Ariostea (Ferrara) [Signatura: Cl. I/105].

${ }^{14}$ Remitimos a R. SEGRE, «Gli ebrei a Pesaro sotto la signoria dei Della Rovere», en Pesaro nell'età dei Della Rovere, III.1 (Venezia 1998) págs. 154-156, y a la contribución muy documentada de A. di Leone LEONI, «Per una storia della nazione portoghese ad Ancona e a Pesaro», en L'Identità dissimulata. Giudaizzanti iberici nell'Europa cristiana dell'età moderna, ed. P. C. I. ZORATTINI (Firenze 2000) págs. 27-97. 
La poesía de Usque reproduce el esquema métrico de la Canción 23 de Canzoniere, que se considera composición juvenil de Petrarca y que con sus ciento sesenta y nueve versos es la más extensa de las incluidas en la magna recopilación del aretino. En lo formal, Usque imita con fidelidad la canción del poeta toscano: coincide el número de versos en cada estrofa (veinte versos, con un único heptasílabo en posición central de la estancia; nueve endecasílabos en el envío final) y respeta con rigor el orden de las rimas. La única diferencia estriba en su extensión: trece estancias, frente a las ocho del modelo.

Este ropaje petrarquista cela un poema compuesto con discreto mérito en el que su autor hace alarde de erudición. Usque enumera y describe las seis edades del mundo, refiriéndose con ellas a cada uno de los períodos de la historia humana según la distribución de Isidoro, y que deriva a su vez de San Agustín: «Aetas autem proprie duobus modis dicitur: aut enim hominis, sicut infantia, iuventus, senectus: aut mundi, cuius prima aetas est ab Adam usque ad Noe; secunda a Noe usque ad Abraham; tertia ab Abraham usque ad David; quarta a David usque ad transmigrationem Iuda in Babyloniam; quinta deinde [a transmigratione Babylonis] usque ad adventum Salvatoris in carne ${ }^{15}$; sexta, quae nunc agitur, usque quo mundus iste finiatur» (Etym. 5.38.5).

Se recordará que en la tradición literaria peninsular el converso Pablo de Santa María (h. 1350-1435), que fue obispo de Burgos, recurrió a parecida distinción cronológica para su composición en coplas de arte mayor Las siete edades del mundo ${ }^{16}$, con la salvedad de que cuanto constituía materia de la tercera edad isidoriana daba pie en su extenso poema a dos momentos históricos diferenciados en los que se agrupaba respectivamente cuanto había ocurrido de

\footnotetext{
${ }^{15}$ Notamos que la materia cristológica, en cambio, estará del todo ausente en el poema de Usque.

16 J. C. CONDE LóPEz analiza y presenta la edición crítica de dicho texto en $L a$ creación de un discurso historiográfico en el cuatrocientos castellano: "Las siete edades del mundo» de Pablo de Santa María, Col. Textos recuperados (Salamanca 1999).
} 
Abraham a Moisés, y acto seguido, en una cuarta edad, a los acontecimientos de mayor relieve desde el reinado de David hasta el cautiverio de los judíos en Babilonia.

La distribución de la materia es irregular en la canción del judío lusitano: una estancia para la primera, segunda, tercera y cuarta edades; cinco estancias para la quinta; cuatro para la sexta. Por lo general, Usque se limita a enumerar los sucesos de mayor relieve en cada uno de estos períodos, aunque se explaya cuando toca una temática que juzga conveniente para su ilustre destinatario. Constituyen, pues, excepción la materia alejandrina (vv. 101-129) y, acto seguido, la narración del apogeo y caída respectiva de los imperios cartaginés y romano (vv. 130-180). El resto de la composición, las últimas cuatro estancias que engloban la actual sexta edad del mundo (vv. 181-260), se reserva en su totalidad al encomio del duque de Urbino, a quien nadie, en toda la historia del mundo, ha superado ni superará jamás hasta el final de los tiempos. Una conclusión que recuerda asimismo el propósito de Pablo de Santa María, quien en las estrofas finales de su extenso poema didáctico había presentado al monarca Juan II como redentor político de Castilla.

Queda poco del dictado petrarquista en el poema de Usque, a no ser el vago recuerdo de la estructura enumerativa medievalizante de los Trionfi. El engarce con su modelo métrico se revela tan sólo en arrancar allí el poeta toscano del recuerdo de su primera edad, cuando éste era aún inmune al reclamo erótico; una edad feliz que se contraponía a la devastación física y mental provocada por los efectos de la pasión sensual que del joven Petrarca se adueñaría años más tarde. Queda también la huella del recurso empleado en el exordium, allí donde el toscano dislocaba el orden sintáctico y demoraba hasta el quinto verso la aparición de la frase principal:

Nel dolce tempo de la prima etade, che nascer vide et anchor quasi in herba

3 la fera voglia che per mio mal crebbe, perché cantando il duol si disacerba, canterò com'io vissi in libertade,

6 mentre Amor nel mio albergo a sdegno s'ebbe. 
Usque extrema dicho procedimiento, pues la cláusula en la que se encierra el sentido de la entera composición no aparecerá sino hasta los versos 181-182: «Né in questa sesta, oh Illustre Guidobaldo!, / unqua fu, né mai fia, chi vi si aguaglie». Y sólo entonces, cuando ya nos hallamos enfrascados en la lectura de la parte final de la canción de Usque, comprendemos la repetición anafórica del complemento circunstancial con el que da inicio el relato de cada una de las edades del mundo (vv. 1, 21, 41, 61, 81 y 181) esbozadas por el lusitano.

La finalidad de este acto poético es clara y Usque la expresa sin reticencias en la decimotercera estancia (vv. 241-260): el autor quisiera poner su ingenio al servicio de Guidobaldo y poder cantar así sus virtudes y su gloria, pero necesita para ello la protección del duque de Urbino. Para la formulación elegante de este ruego, Salomón Usque utiliza la única metáfora feliz del largo poema: su ingenio es una brasa que se extingue («s'ammorza») con lentitud bajo una capa de ceniza; bastaría que alguien la alimentara, añadiéndole combustible («Giungendole materia, a poco a poco»), para que el fuego se avivara y las centellas se elevaran al cielo.

No sabemos si Guidobaldo II correspondió con generosidad a la solicitud del poeta. Sospechamos que no, al menos en la medida en la que éste lo hubiera así deseado cuando concluía su ruego con el anhelo de colocarse al servicio del señor de Urbino: «Un sol suo raggio mi fia scorta e Duca». No hay nada que a mediados de siglo pruebe la estancia de Usque en la corte de Guidobaldo, ni tampoco posteriormente cuando, tras su muerte (28 de septiembre de 1574), le suceda al frente del ducado de Urbino el primogénito Francesco Maria della Rovere.

Editamos por vez primera esta composición italiana, esperando contribuir de este modo a un mejor conocimiento de la biografía y obra de Salomón Usque. Sirva además como estímulo para proseguir en el estudio de la labor intelectual de los judíos peninsulares en su diáspora en Italia, que con frecuencia llevaron a cabo una excelente labor de intermediación entre ambas culturas. 
CANCIÓN DE LAS SEIS EDADES DEL MUNDO ${ }^{17}$

[308r] Canzona dell'Illustrissimo et eccellentissimo Duca d'Urbino.

[309r] Canzona allo Illustrissimo et eccellentissimo Signor Duca d'Urbino.

I. [309r] [La prima età]:

Ne la primiera età non fu in che piacque, da poi che gli elementi hebbe formato

3 l'ethereo cielo e nostra antica madre.

Questo di sole, e luna e stelle ornato, e questa di fior, frondi, herbe e dolci acque,

6 e d'animali di diverse squadre ${ }^{18}$.

Dico che piacque al nostro sommo Padre il lor signor formar. Questo fu l'huomo:

9 primo arbor, primo tronco e primo ramo.

17 Grafías: 5 dolc'acque; 19 ò maledetto; 28 da i nepoti; 43 de i grandi; 49 chebber; 57 co $i$ vivj; 61 Psalmo grapho; 64 succeße; 68 borjoso; 77 laqual; 82 Cyro; 84 dj; 85 RIA; 89 Cyro; 95 ò gran destino; 106 Meloßi; 109 servjtrice; 111 aßomma; 112 Epaminondj, Agesilaj, Platonj; 113 Demostenj; 114 succeßore; 125 divjsero; 127 chogn'un; 130 a l'hora; 132 ogn'una; 137 anzj; 139 Paßate; 156 ogn'un; 167 Ò vero specchio; 177 largho; 181 ò Illustre GUIDOBALDO; 183 ingiustitia; 186 darvj; 187 inapia; 194 dal'asja; 203 SIGNOR; 204 c'havessi; 206 ne i luochj; 206 secretj; 207 retj; 211 dj quelle; 214 ò muta lingua; 215 dannj; 216 ò Alma; 224 salse; 233 regionj de i velocj ventj; 234 Fin che; 234 nubj; 236 intentj; 239 Ò mille; 245 vedraßi; 246 ivj; 250 BRAGIA; 252 SIGNOR; 256 scrivo; 259 ne l'occeano; 261 t en vai; 262 vannj; 265 Annj; 269 DUCA.

[Se han regularizado las grafías de $u / v, j$ postvocálica a final de palabra y el uso de la mayúscula. Se ha acentuado según el uso actual del italiano]

Abreviaturas: DBI: Dizionario Biografico degli Italiani (Roma 1960-); DEI: Carlo BATtista y Giovanni ALESSIO, Dizionario Etimologico Italiano (Firenze 1968); Etym.: San Isidoro DE SEvilla, Etimologías, ed. José OrOz ReTA, Biblioteca de Autores Cristianos 433-434 (Madrid 1982); Pal.: Alonso DE PALENCIA, Universal Vocabulario (Madrid 1967); Tes.: Sebastián DE COVARRuBIAS, Tesoro de la lengua castellana o española, ed. Martín DE RIQUER, Biblioteca. Serie «Lengua y Literatura» 3 (Barcelona, Altafulla 1987).

${ }^{18}$ En un primer momento se escribió «sguadre» y hubo más tarde enmienda. 
Per lo'ntelletto Adamo

chiamollo ${ }^{19}$; e diegli chi il vietato pomo, troppo crudel' altrui, con le man colse, che scacciò loro e noi del Paradiso.

Solcò la terra l'un col corvo aratro;

15 filava l'altra e partoria con latro, d'amaro pianto aspersa gli occhi e'l viso.

Di duo suoi figli, l'uno a Dio si volse

18 e fugli grato, sì ch'invidia tolse

l'altro e l'uccise. Oh maledetto et empio, che desti al mondo d'homicidio essempio!

II. [309v] La seconda:

$21 \quad[N]$ é'n ${ }^{20}$ la seconda, quando il patriarcha (passato'l gran diluvio che'l mondo hebbe per li peccati de suoi primi figli

24 a quai l'audatia con li corpi crebbe) con moglie, e figli, e nuore uscì del'Arca, salvato sol per li divin consigli:

27 che i buoni scampan sempre de' perigli. Empì il mondo il suo seme; e dai nepoti vennero ethiopi, egittii, persi et indi.

30 Non stetter molto d'indi a nascer figli dal ben far remoti: che con il capo lor, Nembrotte audace,

33 fer la superba torre per schivare l'acque, se Dio più n'innondasse il mondo;

${ }^{19}$ Etym. 7.6.4: «Adam, sicut beatus Hieronymus tradit, homo sive terrenus sive terra rubra interpretatur». Hbr. adam: 'hombre' (cf. Tes., s.v. Adán: «en la lengua santa todo hombre se llama Adán, y comprehende ambos sexos»); lat. intellectus: 'sentido, significado' (Pal., s.v.: «lo que se concibe dentro del sentido»).

${ }^{20}$ Suplimos la letra inicial de estrofa, pues al encuadernar el volumen se guillotinaron con descuido los folios del códice. Así también en todas las estrofas pares que ocupan invariablemente el reverso del folio. 
qual confusa mandò poscia ${ }^{21}$ al profondo.

36 In questa età si vide principiare

in Zoroastro l'arte sì fallace ${ }^{22}$,

e 'l regno assirio in chi ruppe la pace;

39 Belo dico io ${ }^{23}$, cui succedendo Nino

nacque Abram, patriarcha peregrino.

III. [310r] La terza:

Men ne la terza etade, in che vivendo

42 il figliuolo d'Abram cominciò il regno

dei grandi argivi nel Peloponeso ${ }^{24}$.

Uscir' gli Hebrei d'Egitto, con il degno

45 capitano che'l mar lor gia partendo,

$o$ ve faraon restò sommerso e preso.

$\mathrm{Fu}$ in Thesaglia il diluvio sì disteso ${ }^{25}$.

48 Cominciar molti regni a nominarsi

dal nome del signor ch'ebber primiero:

Siculo e Ethiope diero

51 nome a Sicilia e a l'Ethiopia. Altri chiamarsi ${ }^{26}$ :

da Boetio, Boetia; da Sardo, Sardigna.

${ }^{21}$ El adverbio ha sido añadido interlinearmente por la misma mano.

22 Etym. 5.39.7 «Zoroastres magicam repperit».

${ }^{23}$ Con el inciso «dico io» tal vez quiere el poeta dejar constancia de que se trata de un dato no consignado por su fuente histórica.

${ }^{24}$ Etym. 5.39.8: «Isaac ann. LX genuit Iacob. Argivorum regnum inchoat».

${ }^{25}$ Etym. 13.22.4: «Tertium diluvium in Thessalia Moysi vel Amphictyonis temporibus fuit, qui tertius post Cecropem regnavit. Cuius temporibus aquarum inluvies maiorem partem populorum Thessaliae absumpsit paucis per refugia montium liberatis, maxime in monte Parnaso; in cuius circuitu Deucalion tunc regno potiebatur, qui tunc ad se ratibus confugientes susceptos per gemina Parnasi iuga fovit et aluit».

${ }^{26}$ Es verso hipermétrico. 
Fiorir ${ }^{27}$ i tenebri. Giason ${ }^{28}$ con gli achivi

54 li colchi del monton privati fenne.

Rappì Helena Paris, e Gretia venne contra Troia acquistar la sua ciprigna.

57 Distrussela, et Enea partì coi vivi verso Italia a rifar la ${ }^{29}$ sua Troia ivi.

Nacque'l pastor per cui solo cadeo

60 col primo sasso quel gran Philisteo ${ }^{30}$.

\section{IV. [310v] La quarta:}

[N]o ne la quarta, quando il psalmographo lo scettro, il trono, et il diadema ${ }^{31}$, e'l manto

63 hebbe, mandando i suoi nemici ad imo.

E gli successe quel che fece il santo edificio e servì la dea di Papho.

66 Non tal destro ${ }^{32}$ architetto com'io stimo, finì in colui che tant'abbracciò il limo (dico in Sardanapalo) il sì borioso ${ }^{33}$

69 imperio assirio e transferisse a' Medi. E cominciaro i $\mathrm{re}^{34} \mathrm{di}$ Macedonia nel regno vittorioso.

72 Didone edificò la gran Cartago.

${ }^{27}$ Se escribió primero «fioriro», enmendado más tarde con el infinitivo.

${ }^{28}$ En el ms. se ha enmendado con una $a$; se diría que en un primer momento se había escrito «Gir son».

29 Se ha enmendado con un trazo separador vertical lo que el copista había escrito en un solo tramo: «rifarla».

${ }^{30}$ La alusión es a David, el cual derrota por sí solo al gigante Goliat.

${ }^{31}$ Parece que se copió primero «diodema» y que posteriormente hubo enmienda de la vocal.

32 Enmendamos lo que en el ms. se transcribió «dentro».

${ }^{33} \mathrm{Ha}$ habido enmienda en el ms. Parece que en un primer momento se copio «boreoso».

${ }^{34} \mathrm{La}(r)$ ha sido reescrita con trazo vigoroso. 
Romolo e Remo fondar dopo questa la Regia Roma, in humil povertade.

75 Furo i suoi cinque re. Fu la cittade Bizantio suo ritratto, hor sì molesta, de la qual Costantin fu tanto vago.

78 Contra Hierusalem venne quel drago Nabuchodonosorre, e la destrusse e seco il preso populo condusse.

V. [311r] La quinta:

81 No ne la quinta, in che la monarchia di persi cominciò sotto il gran Ciro: sì saggio in vincer; nel morir sì sempio.

84 La cui testa, la piena di martiro, satiò di sangue; dicendole: «Ria sangue setisti ${ }^{35}$; et io di sangue t'empio».

87 Uscir gli hebrei di Babilonia e 'l tempio reedificaro. Al'hor Cambise, figlio di Ciro, il regno soggiogò de' persi.

90 E mandaron dispersi i roman, per uscir del fiero artiglio, tutti i lor regi. E poscia governati ${ }^{36}$

93 da consuli. Et, eletti, i primi furo: quel Giulio Brutto e Lutio Colatino. Fioriro in Gretia al'hora, oh gran destino!,

96 Minerva e Marte, uscendo da l'oscuro con infinito numero d'armati.

Contra lor venne Xerse; e già assediati,

99 e per terra e per mar, sol con l'ardire di Themistochle feronli fuggire.

\footnotetext{
${ }^{35}$ La palabra es de muy difícil lectura.

${ }^{36}$ El verbo es elíptico: 'furon poscia governati'.
} 
VI. [311v]:

[F]iorì di Macedonia il regno poscia, 102 sotto il gran padre d'Alessandro il Magno che tributarii gli feo i monti e i piani. Più e più volte vinse con guadagno 105 gli atheniesi; et anco, senza angoscia, i phocesi, i melossi et i thebani.

Vinse gli ilirii, i thessali e i iraniani ${ }^{37}$,

108 li dardani e li scithii. A chi più, in somma, tutta la Gretia si fé servitrice.

Al'hor sola phenice,

111 la qual produce da quei tempi, e assomma: Epaminondi, Agesilai, Platoni, Demosteni, Aristoteli e altri mille.

114 Morto Philippo gli fu successore quel Alessandro che posò in romore l'Asia e si fece le sue terre ancille.

117 Vincendo Dario, quasi havesse sproni, disfè la Persia e diverse regioni.

Da Pella agli Indi dominò nel corso 120 senza porre al desir sfrenato morso.

VII. [312r]:

Poi che colui, che non capea nel mondo, capere in poco luoco il fé la Parcha 123 spogliandol de la sua superba spoglia, il mondo, di cui solo ei fu monarcha, divisero ${ }^{38}$ tra loro, a tondo a tondo, 126 suoi capitani. E chi più può, più spoglia. Nacque discordia e par ch'ognun l'accoglia,

${ }^{37}$ Este último gentilicio resulta de difícil lectura.

${ }^{38}$ Ha habido enmienda del verbo sin que se consiga adivinar la primitiva lectura. 
da quale in tutta l'Asia visse sveglia

129 e in molti parti de l'Europa ancora.

Roma e Cartago al'hora, gonfie d'altezza e di superbia ${ }^{39}$, veglia

132 ognuna a comandare il mondo tutto.

Combattono gli esserciti tra loro;

partorisce al bissogno gli Asdruballi ${ }^{40}$,

135 Cartagine, gli Annoni ${ }^{41}$ e gli Anniballi.

E Roma non produce minor frutto; anzi i Fabii, i Scipioni, a tal lavoro,

138 e i Marcelli, e gli Emilii, e al fin d'alloro,

passate molte guerre, l'alma Roma

de l'Affrica suggetta ornò la chioma.

VIII. [312v]:

$141[\mathrm{~N}]$ on satia anchora di tanta vittoria la sua ingordigia, con le spiegat'ale, invidïosa, move a Gretia guerra.

144 E la soggioga al fin. E poscia assale le richezze de l'Asia; e con gran gloria vince Antioccho e Mitridate atterra.

147 Già fatta sua l'Asia minore, afferra: e Siria, e Palestina, e al fin l'Egitto; e di qua Francia, et Inghilterra, e Spagna,

150 e'l resto d'Alamagna.

Et in breve il mondo fu da lei sconfitto, ministri essendo di cotante imprese:

153 i Cesari, i Pompei, Marii e Metelli,

\footnotetext{
${ }^{39}$ Ha habido enmienda en el ms., donde las dos grafías finales de «superbia» han sido escritas con trazo vigoroso. Antes se había copiado el adjetivo «superba».

${ }^{40}$ Ha habido enmienda en «Asdruballi».

${ }^{41}$ Dos trazos verticales separan en dos tramos «gli Annonj», a menos que se haya cuerido añadir una (h) inicial (Hannonj).
} 
i Luculli et i Scilli, altri infiniti.

Nasce ambition tra lor, nascono liti,

156 che commandare ognun vuol questi e quelli.

Resta a la fin la potentia: è ${ }^{42}$ il paese

in man di Cesar sol senza contese.

159 Quel Giulio Cesar ch'aspiegate insegne

cinquanta due battaglie feo, sì degne.

IX. [313r]:

Costui, costui, ch'in varie imprese vinse

162 un millione con cento e nonanta

duo millia, in anni diece ${ }^{43}$. E dei nemici,

da Bruto e Cassio fu, qual nobil pianta,

165 da ferro svelta. Fortuna il derise, né gli giovaro i fatti sì felici.

Oh vero specchio, in cui specchiar gli amici

168 di questa instabil denno! E non fidarsi

ancorché assisi al colmo di sue rote!

Successegli il nepote

171 Ottaviano, che i nemici sparsi

vinse pel'imprese ${ }^{44}$ che se gli offeriro, ne vendicar ${ }^{45}$ la morte del gran zio.

174 E Brutto, e Cassio, persa ogni battaglia, disperati s'uccisero in Thesaglia, né con morte pagar lor grave fio.

177 Comandò il mondo in largo, in longo e in giro, con pace, senza haver noia o martiro. El tempio del bifronte Giano serra

180 chiaro segnal ch'estinta era ogni guerra.

${ }^{42}$ Hay enmienda donde antes tal vez se había escrito la conjunción «et».

${ }^{43}$ Se ha enmendado con «diece» lo que tal vez se había escrito «dieci».

${ }^{44} \mathrm{La}(\mathrm{p})$ inicial de la preposición se añadió en un segundo momento.

45 Quizás habría que enmendar con «nel vendicar». 
X. [313v] [La sesta]:

[N]é in questa sesta, oh Illustre Guidobaldo!, unqua fu, né mai fia, chi vi si aguaglie ${ }^{46}$

183 in sangue, in arme, in lettre od in giustitia. Se' quanto l'Alma, dentro in sue muraglie, mi mostra col pensier fervido e caldo.

186 Non già per darvi luce, che stoltitia sarebbe e offrir la inopia a la divitia. Mostrar potessi, e a nostri e a strani liti,

189 ragionando ${ }^{47}$ di voi cose direi sì nove che farei aghiacciar gli ethiopi, arder gli scithi.

$192 \mathrm{Ma}$, in somma: che direi, picciolo verme! Se'l nome vostro con sonora tromba, da l'arsa Libia ${ }^{48}$ a l'onde Histre gelate, 195 dove l'Hibero muor, dove l'Eufrate nasce, e più oltre, se si può, rimbomba! $E$ anchor non dice che' ${ }^{49}$ famoso germe ${ }^{50}$ 198 quanto vorria la fama, anzi è in ciò inerme. Quel che narra, minor tant'è dal vero quanto può men la lingua del pensiero.

XI. [314r]:

201 Vorrei pur parte dir de l'infinite virtù ch'a larga man nel vostro petto

${ }^{46}$ Ha habido en el ms. enmienda de lo que se había copiado «aggaglie».

${ }^{47}$ Tras el gerundio se copió el sustantivo «cose», que se borró en un segundo momento.

${ }^{48}$ En el ms. se lee «l'asia Libia»; admitimos la enmienda con el epíteto «arsa» tal como nos sugiere Miguel Requena (UAB).

${ }^{49}$ En un primer momento se escribió «che'l» y luego se enmendó con «del», tal vez pensando en el régimen preposicional corriente (itl. dire di).

50 Tal vez haya que leer «fumoso g.». 
sparser, Signor, tutti i miglior pianeti.

$204 \mathrm{Ma}$ anchor ch'avessi ugual al gran suggetto

lo stile, e non la mia ma mille vite, non perverrei nei luochi lor secreti.

$207 \mathrm{E}$ mi saria più facil corre ${ }^{51}$ in reti

il vento, e annoverar nel ciel le stelle,

e chiuder tutte l'acque in picciol vetro,

210 che poter dir in metro

il sì profondo pelago di quelle.

Ma anchor che sia mia barcha senza speme ${ }^{52}$

213 e segno fatta ai minaccianti scogli ${ }^{53}$, snodati, oh muta lingua, e detta rime che non temano o danni ${ }^{54}$, o morti, o sime.

216 Oh Alma! Tue potentie al regno accogli ${ }^{55}$ !

Svegliati ingegno a le cose supreme, che anchor che morto con voi cadrò insieme!

219 S'avien ch'io spieghi l'ali al bel desio, qual vita aguagliarassi al morir mio? ${ }^{56}$

XII. [314v]:

So, benché grande audatia e questa mia

222 e una maggior di quella di Phetonte, dove la lingua nel parlar si sciolse:

51 Tal vez habría que enmendar con «porre».

52 Verso de lectura problemática: se ha escrito «speme» aprovechando al máximo el breve margen derecho $(s p) \mathrm{y}$, debajo, (eme). Nótese que a la izquierda el copista, o un lector, ha marcado con un calderón el inicio del endecasílabo.

53 En un primer momento se había escrito «ai minaccianti scogli è segno fatta»; el verso fue tachado, se reordenó en el espacio interlinear y se reemplazó.

54 Ha habido enmienda en el ms.: al parecer, un precedente «o dannj», se reemplazó con un poco convincente «o annj» y finalmente se optó por la lección inicial.

55 No es del todo claro si se escribió «accoglie», lo que impediría la rima.

${ }^{56}$ El verso se escribió en el ajustado margen inferior que quedó a disposición del copista, lo que dificulta la lectura. 
s'ardita s'alse, frettolosa smonte.

225 Fatt'era nave che trascorse via, perso remi e timon che "l mar le tolse ${ }^{57}$ -olse

228 che l'aggirano hor quinci, hor quindi intorno.

E'l nocchier per salvarla in van s'adopra.

Ma l'Alma fa in tal opra

231 quel che aquila suol dopo il soggiorno;

che lasciate le terre batte l'ale

a le regioni dei veloci venti

234 finché si asconde in fra le nubi e arriva

in loco $o$ ve spiega la virtù visiva ${ }^{58}$,

tenendo gli occhi suoi fissi et intenti

237 ai bei raggi dil sole. E più non sale,

ma quasi nel mirar si fa immortale ${ }^{59}$.

Oh mille e mille volte alma felice:

240 che gaudio unqua maggior sperar ti lice!

XIII. [315r]:

E lo mio ingegno come bragia stassi

da cenere coperta; che né ${ }^{60}$ luce,

243 né mica di splendor mostra di fuoco.

Son gli effetti ambi in lei, se ben non luce;

ma se la move alcun, chiara vedrassi,

${ }^{57}$ En el margen izquierdo hay unos caracteres cuyo significado no hemos podido desentrañar y que probablemente son signo de un lector que ha notado la ausencia del verso sucesivo.

58 Ante «visiva» nos queda la duda al leer el ms., pues podría leerse «iusiva».

${ }^{59}$ El verso ha sido escrito erróneamente como el penúltimo de la estrofa. Alguien en el margen izquierdo ha numerado los últimos cuatro vv. para facilitar el correcto orden de lectura.

${ }^{60}$ Ha habido enmienda en el ms.; al parecer se había escrito antes «non» en lugar de «né». 
246 e molto più se soffia ivi ${ }^{61}$ un poco.

Giungendole materia, a poco a poco, le scintille salir vedransi al cielo;

249 indi le fiamme anchor con ${ }^{62}$ maggior forza.

La bragia, che s'ammorza, darà splendor se voi con qualche stelo

252 soverrete, Signor, o qualche ${ }^{63}$ duffa ${ }^{64}$. E frutto produrà, com'è il terreno pregno di grano, s'a la sua stagione

255 sente l'acqua, la neve e l'aquilone.

Talché il gran nome vostro al ciel iscrivo, fuor del pensier che'l scioccho vulgo abuffa ${ }^{65}$

258 e là dove il sol nasce e ove s'attuffa: nel'occeano, e a l'orse, e a mezzo l'arco che ne saetta il giorno andarà scarco.

[Congedo] [315v]:

261 Ove te'n vai sì ardita, Canzon mia? Ove è la guida? Dove sono i vanni? Scorgi farfalla il lume che ti acciecca!

264 Non ti turbar, che ben ch'inerme e cieca ${ }^{66}$ tanti monarchi, tanti heroi, tant'anni van meco ch'huopo d'ala non mi fia,

\footnotetext{
${ }^{61}$ Se tachó el adverbio «ivi», que nosotros reintegramos, y se enmendó con la desinencia verbal: -avi («soffiaravi»).

62 Dejamos constancia de enmienda en el ms. para la preposición «con».

${ }^{63}$ Sólo en un segundo momento se añadió «che».

${ }^{64}$ No hemos documentado la voz «duffa», que nos tienta enmendar con itl. stuffa (DEI, s.v., no señala por cierto su entrada en italiano sino hasta el siglo XVI).

${ }^{65} \mathrm{El}$ verbo es de difícil lectura por no haber quedado suficiente espacio al copista en el margen del folio.

${ }^{66}$ No entendemos el porqué de la presencia de un calderón en el margen derecho del verso.
} 
267 e l'almo sol, ove il mio vol s'invia,

forz'è che ovunque egli si giri ${ }^{67}$ luca.

Un sol ${ }^{68}$ suo raggio mi fia scorta e Duca.

Dedicato [a] Vostra Signoria Illustrissima et Eccellentissima

Humile ${ }^{69}$ et devoto servitore

Salamone Usque

${ }^{67}$ Tras el verbo se escribió la preposición «in», que luego se tachó.

${ }^{68}$ Se escribió «suol» en el lugar de «sol», que se enmendó luego con el adverbio.

${ }^{69}$ Se copió «Huimile». 


\section{RESUMEN}

Salomón Usque era hijo de judíos portugueses que la diáspora llevó a Ferrara. Fue tal vez hijo de Abraham Usque, tipógrafo de la Biblia de Ferrara en ladino (1553). Documentamos su paso por Venecia entre 1560 y 1567, alternando con estancias en Constantinopla, la capital otomana en la que probablemente murió. En 1567, el tipógrafo Nicolò Bevilacqua publicó en Venecia su traducción al castellano de la primera parte del Canzoniere de Petrarca. Fue autor de la tragedia L'Ester, refundida por Leone Modena en 1619. Compuso asimismo poesías en lengua italiana. Entre estas últimas registramos una canción encomiástica dirigida a Guidobaldo II Montefeltro (1514-1574), duque de Urbino, incluida en el códice manuscrito N.A. 5 de la Biblioteca Ariostea de Ferrara y que se edita aquí por vez primera. La distribución de la materia enciclopédica remite al modelo remoto de San Isidoro, pero hay analogías con el poema Las siete edades del mundo del converso Pablo de Santa María (h. 1350-1435).

Palabras Clave: Salomón Usque, Guidobaldo II Montefeltro, Ferrara, Pesaro, Pablo de Santa María.

\section{SUMMARY}

Salomón Usque was descendant of Portuguese Jews that reached Ferrara during the Diaspora. He was possibly the son of Abraham Usque typographer of the Ferrara Bible (1553) written in ladino. We have evidence of his passing through Venice between 1560 and 1567, alternating stays in Constantinople, the Ottoman capital where he probably died. We owe to Salomón Usque the translation of Petrarch's Canzoniere into Spanish, published by Nicolò Bevilacqua in Venice in 1567 . Usque is the author of the tragedy titled L'Ester arranged in 1619 by Leone Modena. He also wrote poetry in Italian. Among the manuscripts of the Ferrarian codex N.A. 5 belonging to the Biblioteca Ariostea there is a laudatory canzone addressed to Guidobaldo II Montefeltro (1514-1574), the Duke of Urbino, which is edited for the first time in the present article. The distribution of encyclopedic content reminds the distant source of St. Isidore of Seville, but there are some analogies with the poem Las siete edades del mundo written by Pablo de Santa María (born around 13501435), a Spaniard converted Jew.

KeYwords: Salomón Usque, Guidobaldo II Montefeltro, Ferrara, Pesaro, Pablo de Santa María. 